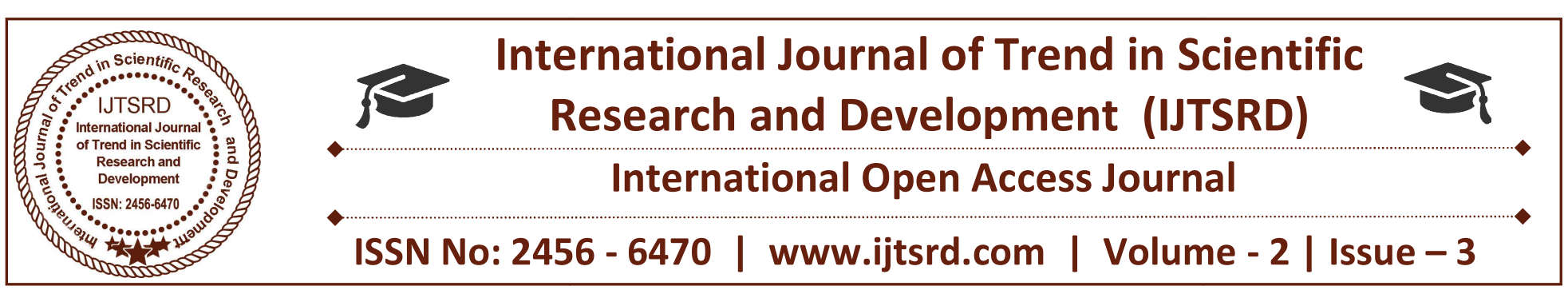

\title{
Design and Development of Vortex Blade less Wind Turbine
}

\author{
Vishal Digambar Bodkhe
}

Keystone School of Engineering, Pune, Maharashtra, India

\begin{abstract}
Vortex-Bladeless is a Spanish SME whose objective is to develop a new concept of wind turbine without blades called Vortex or vorticity wind turbine. This design represents a new paradigm in wind energy and aims to eliminate or reduce many of the existing problems in conventional generators. Due to the significant difference in the project concept, its scope is different from conventional wind turbines. It is particularly suitable for offshore configuration and it could be exploited in wind farms and in environments usually closed to existing ones due to the presence of high intensity winds.

The main objective of this SHAPE project is to develop the needed tools to simulate Fluid-Structure Interaction (FSI) problems and to reproduce the experimental results for scaled models of the VortexBladeless device. In order to do so the Alya code, developed at the Barcelona Supercomputing Center, is adapted to perform the Fluid-Structure Interaction (FSI) problem simulation.
\end{abstract}

Keywords: Fluid-Structure Interaction (FSI), VortexBladeless, vortex induced vibrations (VIV), VortexBladeless

\section{INTRODUCTION}

Fluid-structure interaction problems are multi-physics problems where a fluid interacts with a deformable solid body exerting forces on it. These forces deform the solid body, which modifies the fluid flow as it moves. This kind of interaction is highly complex and different interesting physical phenomena arise. One of them the vortex induced vibrations (VIV), that are vibrations in the solid body resulting from the synchronisation of the vortex shedding frequency and the natural frequencies of vibration of the structure through the so called lock-in effect. This interesting phenomenon has been investigated along years and has been recently used to design new wind energy generators, like the proposed by Vortex-Bladeless.

Vortex-Bladeless [1] is a SME whose objective is to develop a new concept of wind turbine without blades called Vortex or vorticity wind turbine, that uses the VIV. The vortex design aims to eliminate or reduce many of the existing problems in conventional generators and represents a new paradigm of wind energy. It is morphologically simple and it is composed of a single structural component, so its manufacturing, transport, storage and installation have clear advantages.

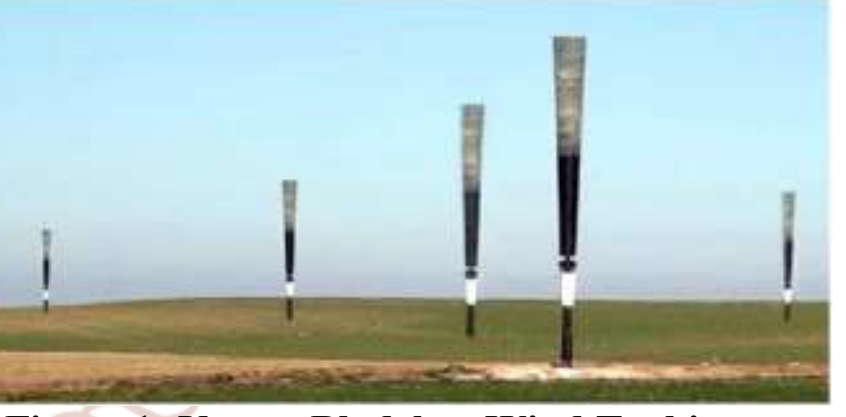

Figure 1: Vortex Bladeless Wind Turbine

Moreover, the numerical simulation of such problems is challenging and has received a lot of attention in the past few years. Different approaches and techniques exist to solve the FSI problems numerically and can be useful to simulate the VIV phenomena coming from the Vortex-Bladeless device. One of these approaches is the staggered multi-code one, where a code is in charge of the fluid flow simulation and another code is in charge of the solid dynamics simulation while the interaction among them comes through the exchange of information of boundary conditions and surface forces. In the present project 
the Alya code, developed at the Barcelona Supercomputing Center, is adapted and used to perform the Fluid-Structure Interaction (FSI) problem simulation for a scaled experimental vortex-bladeless device, and a comparison between the numerical and experimental results is done.

\section{LITERTURE SURVEY}

Today, India is stepping towards becoming a global super power. This implies that, it is leading the list of developing countries in terms of economic development. Hence its energy requirement is going to increase manifold in the coming decades. To meet its energy requirement, coal cannot be the primary source of energy. This is because coal is depleting very fast. It is estimated that within few decades coal will get exhausted. The next clean choice of energy is solar power, but due to its lower concentration per unit area, it is very costly. Many authors suggested many different ideas on this, and some of them are as:

Vortex-Induced Vibrations (VIVs) of a circular cylinder are analyzed as a potential source for energyharvesting. To this end, VIV is described by a one-degreeof-freedom model where fluid forces are introduced from experimental data from forced vibration tests. The analysis reveals that encouraging high efficiency values can be achieved for high Reynolds numbers [1].

As the regions with high wind speed are limited, the installation of conventional windmill is limited. Windmills that would provide safe, quite, simple, affordable and work on lesser wind speeds are need of the hour[5]. The Bladeless Windmill is such a concept which works on the phenomenon of vortex shedding to capture the energy produced. Generally, structures are designed to minimize vortex induced vibrations in order to minimize mechanical failures [6]. But to increase the vibrations in order to convert vortex induced vibrations into electricity, the study provides the scope and feasibility of the bladeless windmill [2]. To develop a compact device that is able to harvest wind energy and transform it into electrical energy using the concept of vortex shedding is proposed by Correa .R . When calibrated correctly, the vortex shedding will induce resonant oscillation. Electricity would be collected from this oscillation using a magnet and coil assembly. This method was proven to work in water, but has not been applied to air currents. The results demonstrate a potential for vortex induced vibration to be utilized with wind to create electricity, however it will be difficult due to the low density of air compared to other fluid mediums, such as water [3].

\section{PROPOSED WORK}

The non-linear resonance phenomenon known as Vortex-Induced Vibration (VIV) has much relevance in several branches of mechanical engineering. For example, it can be observed in civil structures, like slender chimneys stacks, tall buildings, electric power lines or bridges, to name a few. It is also usual in offshore structures or in the tubes of heat exchange devices. Because its practical and scientific interest, VIV has lead to a large number of fundamental studies. Usually, VIV is considered as an undesirable effect, as it may seriously affect the structural integrity or the reliability of performance, but along this report we will see that if the vibration is substantial, it can be used to extract useful energy from the surrounding flow.

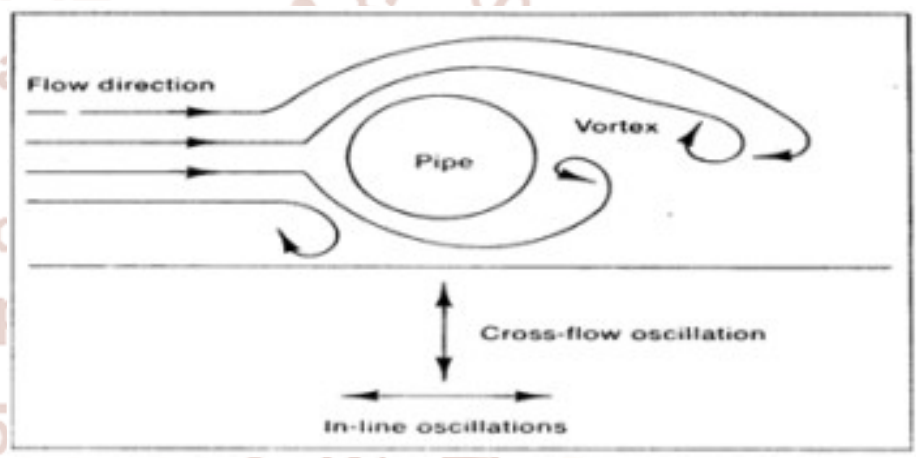

Figure 2: Vortex Induced Vibration

When a fluid flows toward the leading edge of a bluff body, the pressure in the fluid rises from the free steam pressure to the stagnation pressure. When the flow speed is low, i.e. the Reynolds number is low, pressure on both sides of the bluff body remains symmetric and no turbulence appears. When the flow speed is increased to a critical value, pressure on both sides of the bluff body becomes unstable, which causes a regular pattern of vortices, called vortex street or Kármán vortex street. Certain transduction mechanisms can be employed where vortices happen and thus energy can be extracted. This method is suitable both air flow and liquid flow.

Vortex shedding is a widely occurring phenomenon applicable to nearly any bluff (nonstreamlined) body submerged in a fluid flow. Since any real fluid flow is viscous, there will be a significant boundary layer on 
the bodies' surface for all but the lowest Reynolds number flows. At some point along the bodies' surface, separation of the boundary layer will occur, depending on the exact surface geometry. This separated layer, which bounds the wake and free stream, will tend to cause fluid rotation, since its outer side, in contact with the free stream, moves faster than its inner side, in contact with the wake. It is this rotation which then results in the formation of individual vortices, which are then shed from the rear of the body and travel down the wake. Typically, a pattern of periodic, alternating vortex shedding will occur in the flow behind the body, which is referred to as a vortex street. Depending on the characteristics of the flow, mainly the Reynolds number, different types of vortex streets may form, which will be discussed later in more detail. When the pattern of shed vortices is not symmetrical about the body, which is the casein any vortex street, an irregular pressure distribution is formed on the upper and lower sides of the body, which results in a net lift force perpendicular to the flow direction. Since the vortices are shed in a periodic manner, the resulting lift forces on the body also vary periodically with time, and there for can induce oscillatory motion of the body.

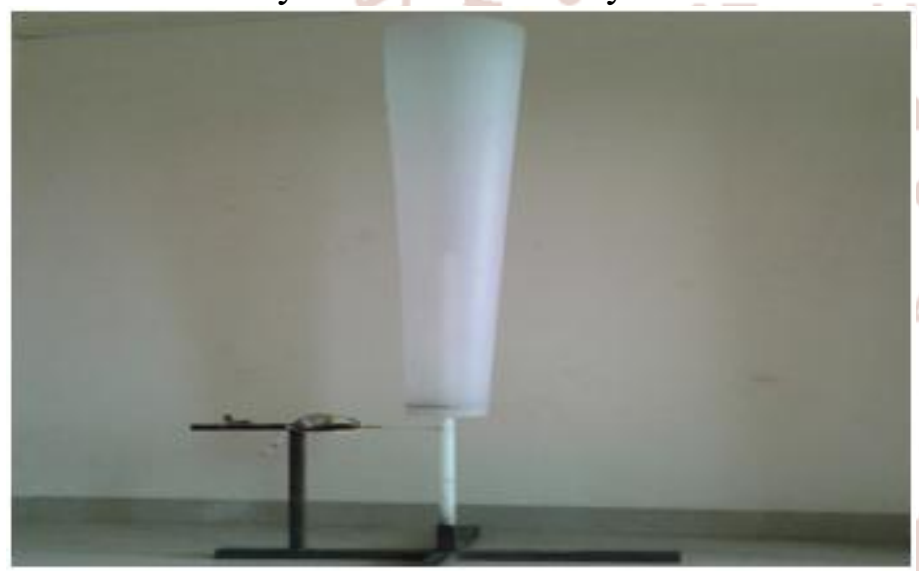

Figure 3: Construction Of Vortex Bladeless Wind Turbine Power Generation

One simple structure with following components as-

1. Rod: The rod gives a strength and flexibility to the moment while minimizing energy dissipation.

2. Generation System: The kinetic energy from the wind is converted into the electricity by using motor, which is attached to the rack and pinion mechanism.

3. Mast: It's a light circular section structure made up of fibre glass and carbon fibre. The mast act as a wind breaker that generates the oscillatory motion

Instead of tuning what the turbine does is oscillate there by producing moment and displacement. The system is based on the same principle as an alternator electromagnetic induction. Then multiply that moment and speed magnetically without use of gear assemblies or ball bearing and that is the mechanical energy of the structure into electricity.

What happens is that structure attracts whirl winds which develop at determined frequencies depending on the speed at which the wind is travelling when the frequency of the whirl wind or vortex coincides with the frequency of the structure. It's than that energy is absorbed reaching a peak due to aeroelastic coupling. It's a great way to transmitting energy from a fluid source to a structure.

\section{CONCLUSION}

The Alya code was adapted in order to simulate the physical response of a scaled device of the VortexBladeless wind energy generator. The simulation was done using a multi-code coupling approach with a loose coupling algorithm. The comparison between the numerical results and the experimental data provided by the SME is very good, with maximum relative errors of less than $10 \%$. This SHAPE project has undoubtedly approached the SME to the HPC and provided an excellent framework to achieve the obtained results.

\section{REFERENCES}

1. Antonio Barrero-Gil, Santiago Pindado, Sergio Avila; Extracting energy from Vortex-Induced Vibrations: A parametric study; Universidad Politecnica de Madrid, Plaza Cardenal Cisneros 3, E-28040 Madrid, Svain

2. SauravBobde,SameerJadhav, Study of Vortex Induced Vibrations for Harvesting Energy; IJIRST - International Journal for Innovative Research in Science \& Technology| Volume 2 | Issue 11 | April 2016

3. Robert Correa,EricCremer,Wind harvesting via Vortex Induced vibration; BJS-WD14.

4. J.C.Cajas,D.J.Yanej;SHAPE Project Vortex Bladeless:Parallel multi-code coupling for fluid structure interaction in wind energy.

5. www.prace-ri.eu

6. www.vortexbladeless.com

7. J.C. Cajas, M. Zavala, G. Houzeaux, E. Casoni, M. Vázquez, C. Moulinec, and Y. Fournier. Fluid Structure Interaction in HPC Multi-Code coupling. In The Fourth International Conference on Parallel, Distributed, Grid and Cloud Computing for Engineering (PARENG15), Dubrovnik (Croatia), 24-27 March 2015. 